\title{
New Look at Nonlinear Aerodynamics in Analysis of Hypersonic Panel Flutter
}

\author{
Dan Xie, Min Xu, Honghua Dai, and Tao Chen \\ College of Astronautics, Northwestern Polytechnical University, Xian 710072, China \\ Correspondence should be addressed to Honghua Dai; hhdai@nwpu.edu.cn
}

Received 25 November 2016; Revised 13 February 2017; Accepted 28 February 2017; Published 23 March 2017

Academic Editor: Xiao-Qiao He

Copyright (C) 2017 Dan Xie et al. This is an open access article distributed under the Creative Commons Attribution License, which permits unrestricted use, distribution, and reproduction in any medium, provided the original work is properly cited.

\begin{abstract}
A simply supported plate fluttering in hypersonic flow is investigated considering both the airflow and structural nonlinearities. Third-order piston theory is used for nonlinear aerodynamic loading, and von Karman plate theory is used for modeling the nonlinear strain-displacement relation. The Galerkin method is applied to project the partial differential governing equations (PDEs) into a set of ordinary differential equations (ODEs) in time, which is then solved by numerical integration method. In observation of limit cycle oscillations (LCO) and evolution of dynamic behaviors, nonlinear aerodynamic loading produces a smaller positive deflection peak and more complex bifurcation diagrams compared with linear aerodynamics. Moreover, a LCO obtained with the linear aerodynamics is mostly a nonsimple harmonic motion but when the aerodynamic nonlinearity is considered more complex motions are obtained, which is important in the evaluation of fatigue life. The parameters of Mach number, dynamic pressure, and in-plane thermal stresses all affect the aerodynamic nonlinearity. For a specific Mach number, there is a critical dynamic pressure beyond which the aerodynamic nonlinearity has to be considered. For a higher temperature, a lower critical dynamic pressure is required. Each nonlinear aerodynamic term in the full third-order piston theory is evaluated, based on which the nonlinear aerodynamic formulation has been simplified.
\end{abstract}

\section{Introduction}

With the increase of the flight speed of modern flight vehicles, panel flutter, a localized aeroelastic problem representing a small portion of the skin on the surface of hypersonic vehicles, is attracting more and more attentions. Many researches have been done in the analysis of panel flutter in supersonic flow $(\mathrm{Ma}<5)$ using the linear aerodynamic theory such as the first-order piston theory [1-5] or linearized potential flow theory [6-8]. Particularly, Carrera and his coworkers applied a finite element structural model coupling with first-order piston theory for aerodynamic model to calculate the flutter boundaries of curved panels [9] and versatile thermal insulation (VTI) panels with pinched boundary conditions [10, 11].

When a panel is fluttering at hypersonic speed $(\mathrm{Ma}>$ 5 ), the nonlinearities involved with panel flutter arise from both the structural and aerodynamic models [12, 13]. Aerodynamic nonlinearity was first considered in conjunction with structural nonlinearity by McIntosh Jr. et al. [14-16] in analysis of simply supported panels fluttering in hypersonic flow. Two nonlinear aerodynamic terms, $(\partial w / \partial x)^{2}$ and $(\partial w / \partial x)(\partial w / \partial t)$, taken from the third-order piston theory, were added to the linear piston theory to account for the aerodynamic nonlinearity [17]. Using the finite element method, Gray and his coworkers $[18,19]$ presented the large-amplitude LCO with the full third-order piston theory to assess the influence of aerodynamic nonlinearity on the hypersonic panel flutter.

The aforementioned studies suggest that (1) the nonlinear aerodynamic loading pushes the panel into the cavity due to the overpressure from the additional nonlinear aerodynamic terms and (2) the nonlinear aerodynamic loading acts to increase panel deflection as "soft spring," which decreases the bending stiffness of the fluttering panel $[20,21]$. To the contrary, the nonlinear membrane stress due to the structural nonlinearity serves as "hard spring," which increases the bending stiffness of the fluttering panel $[22,23]$ and (3) the interplay between the nonlinear mechanisms of "soft spring" 


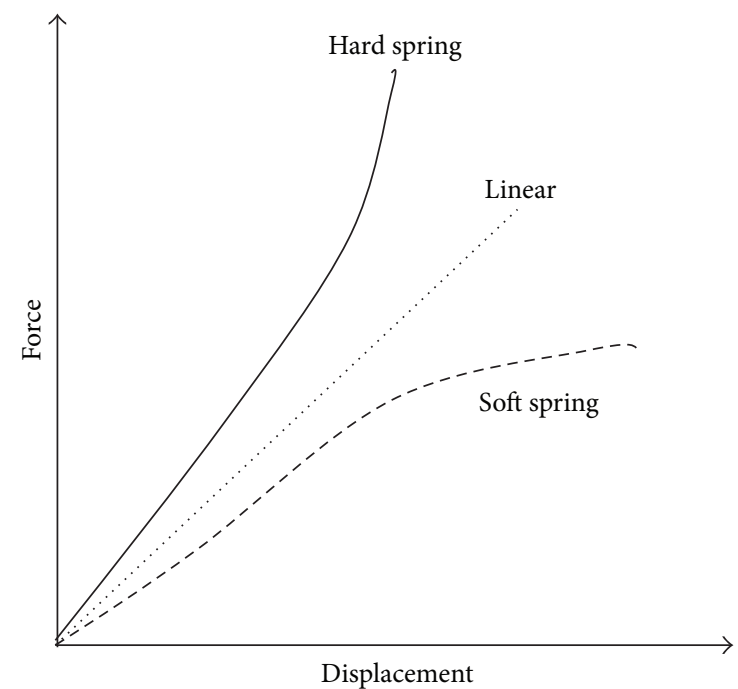

FIGURE 1: Effect of "soft spring" and "hard spring" considering aerodynamic and structural nonlinearities.

and "hard spring" distinguishes the panel flutter at hypersonic speeds from that at supersonic speeds. Specially, the "soft spring" and "hard spring" are demonstrated simply in Figure 1. The existing literature, however, is mainly concerned with the influence of aerodynamic nonlinearity on the LCO amplitude, and Mach number satisfying $\mathrm{Ma}>5$ is the only criterion for applying the nonlinear aerodynamic theory.

In the present study, a simply supported square plate fluttering in hypersonic flow is considered. In addition to the LCOs, more complex motions such as quasi-periodic and chaotic motions are observed when the aerodynamic nonlinearity has been considered. The effects of Mach number, dynamic pressure, and temperature on the aerodynamic nonlinearity are investigated. The influence of aerodynamic nonlinearity on the nonlinear dynamical response of the panel is evaluated via comparing the nonlinear (third-order) piston theory with the linear (first-order) one.

The remainder of this paper is organized as follows. In Section 2, the aeroelastic equations of motion of fluttering panel with von Karman plate theory and both the linear and nonlinear aerodynamic piston theories are formulated. The effects of parameters such as Mach number, dynamic pressure, and temperature on aerodynamic nonlinearity are discussed in Section 3. LCO deflection and bifurcation diagrams versus dynamic pressure are both observed using the linear and nonlinear aerodynamic loadings. The effect of each nonlinear aerodynamic term in the full third-order piston theory is also evaluated thoroughly. Finally, some conclusions are drawn in Section 4.

\section{Theoretical Analysis}

2.1. Aeroelastic Equations. For a simply supported plate, considering the inertial force, aerodynamic force, nonlinear membrane stresses due to the large deflection, and uniform in-plane thermal stresses $N_{x}^{T}$ and $N_{y}^{T}$, the aeroelastic equations can be written as follows:

$$
\begin{aligned}
D( & \left.\frac{\partial^{4} w}{\partial x^{4}}+2 \frac{\partial^{4} w}{\partial x^{2} \partial y^{2}}+\frac{\partial^{4} w}{\partial y^{4}}\right) \\
= & \left(\frac{\partial^{2} \Phi}{\partial y^{2}}+N_{x}^{T}\right) \frac{\partial^{2} w}{\partial x^{2}}+\left(\frac{\partial^{2} \Phi}{\partial x^{2}}+N_{y}^{T}\right) \frac{\partial^{2} w}{\partial y^{2}} \\
& -2\left(\frac{\partial^{2} \Phi}{\partial x \partial y}\right) \frac{\partial^{2} w}{\partial x \partial y}-\left(p-p_{\infty}\right)-\rho_{m} h \frac{\partial^{2} w}{\partial t^{2}}, \\
\frac{1}{E h}( & \left(\frac{\partial^{4} \Phi}{\partial x^{4}}+2 \frac{\partial^{4} \Phi}{\partial x^{2} \partial y^{2}}+\frac{\partial^{4} \Phi}{\partial y^{4}}\right) \\
= & \left(\frac{\partial^{2} w}{\partial x \partial y}\right)^{2}-\frac{\partial^{2} w}{\partial x^{2}} \frac{\partial^{2} w}{\partial y^{2}},
\end{aligned}
$$

where the thermal stresses are given by the quasi-steady thermal stress theory

$$
N_{x}^{T}=N_{y}^{T}=-\frac{E h \alpha T}{(1-\nu)} .
$$

$p-p_{\infty}$ is aerodynamic pressure, which will be modeled with both the first-order and third-order piston theories. The firstorder piston theory is a linear formulation in terms of $\partial w / \partial x$ and $\partial w / \partial t$ :

$$
p-p_{\infty}=\frac{2 q}{\beta}\left[\frac{\partial w}{\partial x}+\left(\frac{\mathrm{Ma}^{2}-2}{\mathrm{Ma}^{2}-1}\right) \frac{1}{U} \frac{\partial w}{\partial t}\right] .
$$

For $\mathrm{Ma} \gg 1,\left(\mathrm{Ma}^{2}-2\right) /\left(\mathrm{Ma}^{2}-1\right) \rightarrow 1$. By contrast, the third-order piston theory considering the aerodynamic nonlinearity includes the nonlinear terms of $(\partial w / \partial x)^{2},(\partial w / \partial t)^{2}$, $(\partial w / \partial x)^{3}$, and $(\partial w / \partial t)^{3}$,

$$
\begin{gathered}
p-p_{\infty}=\frac{2 q}{\beta}\left\{\left[C_{1 t} \frac{1}{U} \frac{\partial w}{\partial t}+C_{1 x} \frac{\partial w}{\partial x}\right]\right. \\
+\frac{\gamma+1}{4} \mathrm{Ma}\left[C_{2 t} \frac{1}{U} \frac{\partial w}{\partial t}+C_{2 x} \frac{\partial w}{\partial x}\right]^{2} \\
\left.+\frac{\gamma+1}{12} \mathrm{Ma}^{2}\left[C_{3 t} \frac{1}{U} \frac{\partial w}{\partial t}+C_{3 x} \frac{\partial w}{\partial x}\right]^{3}\right\},
\end{gathered}
$$

where $C_{i t}, C_{i x}(i=1,2,3)$ are parameters with values of 0 or 1 for evaluating the influence of each nonlinear aerodynamic term. 
The aerodynamic pressure with the first-order piston theory is taken as an example, substituting (3) and (4) into (1) and (2), using appropriate nondimensionalization

$$
\begin{aligned}
\xi & \equiv \frac{x}{a}, \\
\eta & \equiv \frac{y}{b}, \\
W & \equiv \frac{w}{h}, \\
\lambda & \equiv \frac{2 q a^{3}}{\beta D}, \\
\mu & \equiv \frac{\rho a}{\rho_{m} h}, \\
\tau & \equiv t\left(\frac{D}{\rho_{m} h a^{4}}\right)^{1 / 2}, \\
R_{x(y)}^{T} & =\frac{N_{x}^{T} a^{2}}{D}=\frac{N_{y}^{T} a^{2}}{D} \equiv-12(1+\nu)\left(\frac{a}{h}\right)^{2} \alpha T
\end{aligned}
$$

and then we can yield the nondimensional aeroelastic equations:

$$
\begin{aligned}
\frac{\partial^{4} W}{\partial \xi^{4}} & +\frac{2 a^{2}}{b^{2}} \frac{\partial^{4} W}{\partial \xi^{2} \partial \eta^{2}}+\frac{a^{4}}{b^{4}} \frac{\partial^{4} W}{\partial \eta^{4}} \\
= & \left(\frac{a^{2}}{D b^{2}} \frac{\partial^{2} \Phi}{\partial \eta^{2}}+R_{x}^{T}\right) \frac{\partial^{2} W}{\partial \xi^{2}} \\
& +\left(\frac{a^{2}}{D b^{2}} \frac{\partial^{2} \Phi}{\partial \xi^{2}}+\frac{a^{4}}{b^{4}} R_{y}^{T}\right) \frac{\partial^{2} W}{\partial \eta^{2}}-\frac{2 a^{2}}{D b^{2}} \frac{\partial^{2} \Phi}{\partial \xi \partial \eta} \frac{\partial^{2} W}{\partial \xi \partial \eta} \\
& -\lambda\left[\left(\frac{\mu}{\beta \lambda}\right)^{1 / 2} \frac{\partial W}{\partial \tau}+\frac{\partial W}{\partial \xi}\right]-\frac{\partial^{2} W}{\partial \tau^{2}}, \\
\frac{\partial^{4} \Phi}{\partial \xi^{4}} & +\frac{2 a^{2}}{b^{2}} \frac{\partial^{4} \Phi}{\partial \xi^{2} \partial \eta^{2}}+\frac{a^{4}}{b^{4}} \frac{\partial^{4} \Phi}{\partial \eta^{4}} \\
= & \frac{E h^{3} a^{2}}{b^{2}}\left[\left(\frac{\partial^{2} W}{\partial \xi \partial \eta}\right)^{2}-\frac{\partial^{2} W}{\partial \xi^{2}} \frac{\partial^{2} W}{\partial \eta^{2}}\right] .
\end{aligned}
$$

2.1.1. The Galerkin Method. For a simply supported threedimensional plate, an approximation for the panel shape can be expressed with a set of bisine functions [24-26], which automatically satisfies the support conditions,

$$
W(\xi, \eta, \tau)=\sum_{m=1}^{M} a_{m}(\tau) \sin (m \pi \xi) \sin (\pi \eta) .
$$

Here only the first spanwise mode is retained according to Dowell's work in 1966 [1], because compared to the bending in chordwise, the bending in spanwise is much smaller. In addition, the number of modes of panel in chordwise $(x$ direction), $M$, can be determined via divergence analysis, which has been done in the authors' previous work [3]; hence, here $M$ is chosen directly.

Based on the expansion in (9), along with the boundary conditions, the general and particular solutions for the Airy stress $\Phi$ can be calculated [27-29]. Then by multiplying (7) with each basis function $\sin (r \pi \xi), \sin (\pi \eta)$ retained in (9) $(r=1, \ldots, M)$ and integrating along the panel length and width, a set of $M$ second-order ODEs will be obtained for the coefficients $a_{m}(\tau)$ of the harmonic modes. For the sake of brevity, only the resulting reduced order ODEs are displayed as follows:

$$
\begin{aligned}
\frac{d^{2} a_{r}}{d \tau^{2}} & =-a_{r} \pi^{4}\left[r^{2}+\left(\frac{a}{b}\right)^{2}\right]^{2} \\
- & \lambda\{\underbrace{\left(\frac{\mu}{\beta \lambda}\right)^{1 / 2} \frac{d a_{r}}{d \tau}+\sum_{m}^{M} \frac{2 r m}{r^{2}-m^{2}}\left[1-(-1)^{r+m}\right] a_{m}}_{*}\} \\
& -3 \pi^{4}\left(1-v^{2}\right) \\
& \cdot\left\{\frac{a_{r}}{2}\left[r^{2} A+\left(\frac{a}{b}\right)^{2} B\right]+\left(\frac{a}{b}\right)^{4}\left[C+D+\frac{E}{4}-\frac{F}{2}\right]\right\} \\
& -R_{x}^{T} \pi^{2} r^{2} a_{r}-R_{y}^{T}\left(\frac{a}{b}\right)^{2} \pi^{2} a_{r}+\frac{8 P}{\pi^{2}} \frac{\left[1-(-1)^{r}\right]}{r} .
\end{aligned}
$$

When using the third-order piston theory, the aerodynamic part denoted by " $\star$ " contains seven additional nonlinear aerodynamic terms

$$
\begin{aligned}
C_{1 t} & \left(\frac{\mu}{\beta \lambda}\right)^{1 / 2} \frac{d a_{r}}{d \tau}+C_{1 x} \sum_{m}^{M} 2 m a_{m} \zeta_{r, m}+\frac{\gamma+1}{4} \mathrm{Ma}\left\{\frac{8}{3 \pi^{2}} C_{2 t}^{2} \frac{\mu}{\beta \lambda} \sum_{m}^{M} \sum_{n}^{M} \frac{d a_{m}}{d \tau} \frac{d a_{n}}{d \tau}\left(\zeta_{r, m-n}-\zeta_{r, m+n}\right)\right. \\
& \left.+\frac{8}{3} C_{2 t} C_{2 x}\left(\frac{\mu}{\beta \lambda}\right)^{1 / 2} \sum_{m}^{M} \sum_{n}^{M} n \frac{d a_{m}}{d \tau} a_{n}\left(\delta_{r, m+n}+\delta_{r, m-n}\right)+\frac{8}{3} C_{2 x}^{2} \sum_{m}^{M} \sum_{n}^{M} m n a_{m} a_{n}\left(\zeta_{r, m+n}+\zeta_{r, m-n}\right)\right\}+\frac{\gamma+1}{12} \\
& \cdot \mathrm{Ma}^{2}\left\{\frac{3}{16} C_{3 t}^{3}\left(\frac{\mu}{\beta \lambda}\right)^{3 / 2} \sum_{m}^{M} \sum_{n}^{M} \sum_{s}^{M} \frac{d a_{m}}{d \tau} \frac{d a_{n}}{d \tau} \frac{d a_{s}}{d \tau}\left(\delta_{m+n, r+s}-\delta_{m+n, r-s}+\delta_{m-n, r-s}-\delta_{m-n, r+s}\right)\right. \\
& +\frac{3 \pi^{2}}{8} C_{3 x}^{3} \sum_{m}^{M} \sum_{n}^{M} \sum_{s}^{M} m n s a_{m} a_{n} a_{s}\left(\zeta_{r+s, m+n}+\zeta_{r+s, m-n}+\zeta_{r-s, m+n}+\zeta_{r-s, m-n}\right) \\
& +\frac{9}{8} C_{3 t}^{2} C_{3 x} \frac{\mu}{\beta \lambda} \sum_{m}^{M} \sum_{n}^{M} \sum_{s}^{M} s \frac{d a_{m}}{d \tau} \frac{d a_{n}}{d \tau} a_{s}\left(\zeta_{r+s, m-n}-\zeta_{r+s, m+n}+\zeta_{r-s, m-n}-\zeta_{r-s, m+n}\right) \\
& \left.+\frac{9 \pi^{2}}{16} C_{3 t} C_{3 x}^{2}\left(\frac{\mu}{\beta \lambda}\right)^{1 / 2} \sum_{m}^{M} \sum_{n}^{M} \sum_{s}^{M} n s \frac{d a_{m}}{d \tau} a_{n} a_{s}\left(\delta_{m+n, r+s}+\delta_{m+n, r-s}+\delta_{m-n, r+s}+\delta_{m-n, r-s}\right)\right\},
\end{aligned}
$$




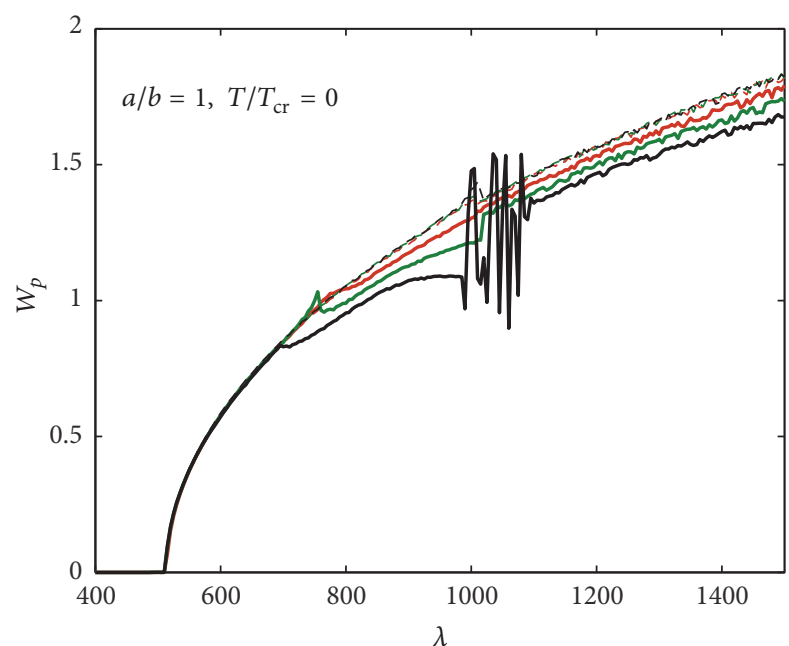

Red line: $\mathrm{Ma}=5$ Green line: $\mathrm{Ma}=10$ Black line: $\mathrm{Ma}=15$
Dash line: 1st-order piston Solid line: 3rd-order piston

(a)

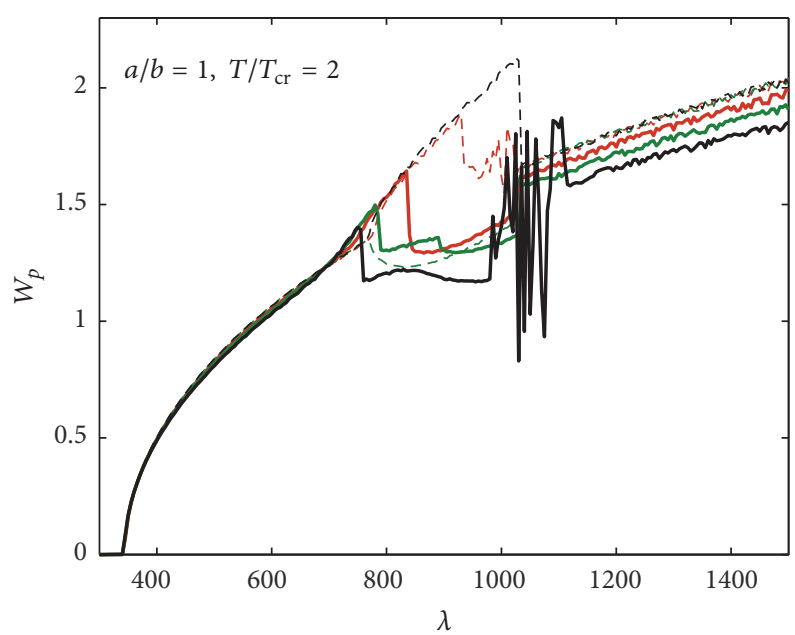

Red line: $\mathrm{Ma}=5$ Green line: $\mathrm{Ma}=10$ Black line: $\mathrm{Ma}=15$
Dash line: 1st-order piston Solid line: $3 r d-o r d e r$ piston

(b)

FIGURE 2: Positive LCO deflection peak versus dynamic pressure for a square panel at Ma =5, 10, and 15 at different temperatures: (a) $T / T_{\text {cr }}=0 ;$ (b) $T / T_{\text {cr }}=2$.

where

$$
\begin{gathered}
\zeta_{p, q}=\frac{p\left[1-(-1)^{p+q}\right]}{p^{2}-q^{2}}, \\
\delta_{p, q}= \begin{cases}0, & p \neq q, \\
1, & p=q .\end{cases}
\end{gathered}
$$

The expressions for coefficients of $A-F$ can be found in [1]. We can solve (10) by the direct time integration method [30, 31].

\section{Results and Discussions}

In this section, a simply supported square plate in supersonic flow is explored, with the material properties and geometrical dimensions as $E=71 \mathrm{GPa}, v=0.3, \alpha=2.34 \times 10^{-6} /{ }^{\circ} \mathrm{C}$, $\rho_{m}=2750 \mathrm{~kg} / \mathrm{m}^{3}$, and $h / a=1 / 300$. The flow density is $\rho=$ $0.413 \mathrm{~kg} / \mathrm{m}^{3}$. All figures are plots at a typical point $\xi=0.75$, $\eta=0.5$. A critical temperature $T_{\mathrm{cr}}=\pi^{2} h^{2} /\left[12(1+v) \alpha a^{2}\right]$ is defined.

3.1. Observation of LCO with Linear and Nonlinear Piston Theories. At lower temperatures of $T / T_{\mathrm{cr}}=0$ and $T / T_{\mathrm{cr}}=$ 2 shown in Figures 2(a) and 2(b), respectively, the panel undergoes the LCO motions in a range of dynamic pressure of interest, and the curves in terms of panel positive deflection peak versus dynamic pressure at Mach number of 5, 10, and 15 are presented. The curves of $+W_{p}$ versus $\lambda$ from the linear aerodynamic theory are very close for various Mach number. By contrast, considering the aerodynamic nonlinearity, the curves tend to be below the ones using the linear aerodynamic theory, and a higher Mach number produces a lower curve. With the increase of dynamic pressure, the deviation between the linear and nonlinear aerodynamic theories increases first and then decreases. Particularly, when $\lambda=1000 \sim 1100$ for Ma $=15$, the peak oscillates violently, which is actually because the panel oscillates with changeable deflection peaks rather than a definite one like LCO. The physical phenomenon is truly where the panel oscillates in a complex motion like quasiperiodic or chaos instead of a simple harmonic oscillation, which is due to the higher nonlinear aerodynamics with 3rdorder piston theory under high Mach number.

Comparing Figures 2(a) and 2(b), the aerodynamic nonlinearity is augmented with the increase of temperature. Specifically, for $750<\lambda<1100$ in Figure 2(b), the nonlinear aerodynamic loading produces totally different results. In Figure 2(b), the responses under two specific dynamic pressures of $\lambda=900$ and $\lambda=1000$ at $\mathrm{Ma}=15$ are considered to make the comparisons easily visible. As shown in Figures 3(a), 3(c), and 3(e) and Figures 3(b), 3(d), and $3(\mathrm{f})$, the phase portraits, deflection shapes, and stress distributions in $x$ direction for $\lambda=900$ and $\lambda=1000$, respectively, using the linear and nonlinear aerodynamics, are presented. The results show that, at $\lambda=900$, the dynamic motions with the linear and nonlinear aerodynamic theories are both LCO motions shown in Figure 3(a), but the positive deflection peak is reduced and the negative deflection peak is increased. The deflection shapes in Figure 3(c) are similar but with different amplitudes. The nonlinear aerodynamic loading has a larger influence on the stress distribution as shown in Figure 3(e). By contrast, for $\lambda=1000$ shown in Figure 3(b), the nonlinear aerodynamic theory obtains 

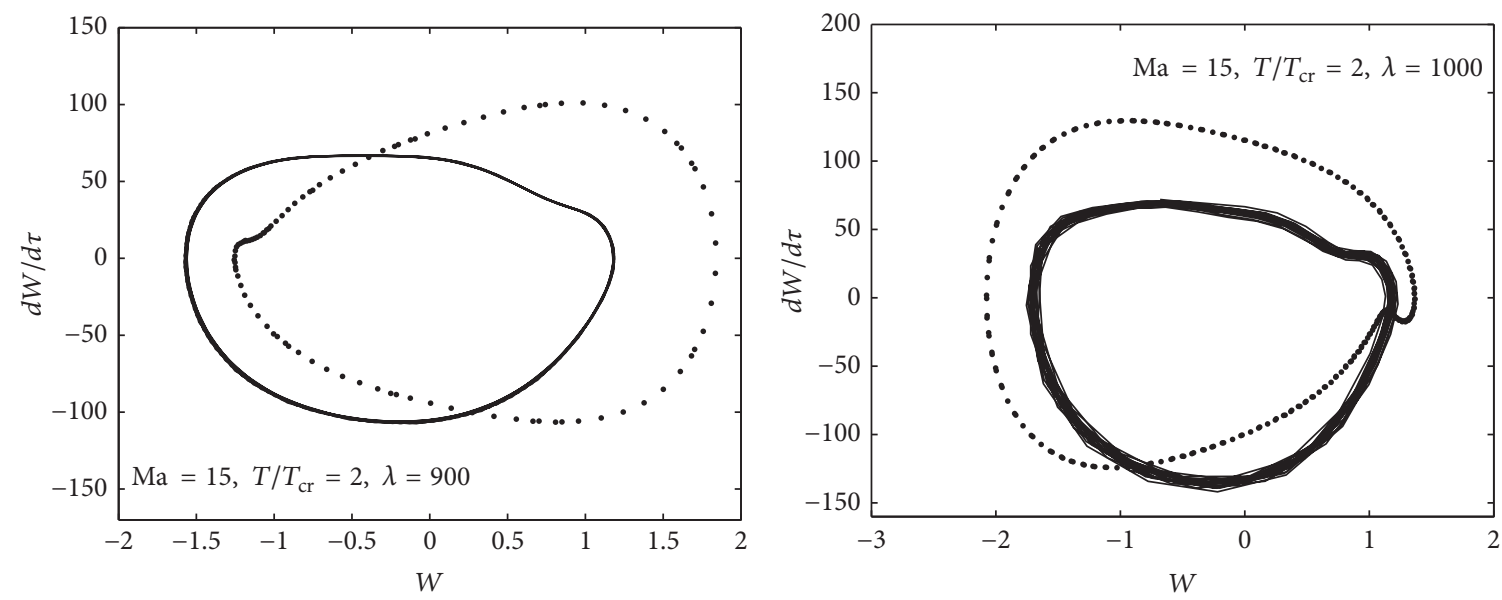

- 1st-order piston

- 1st-order piston

- 3rd-order piston

(a)

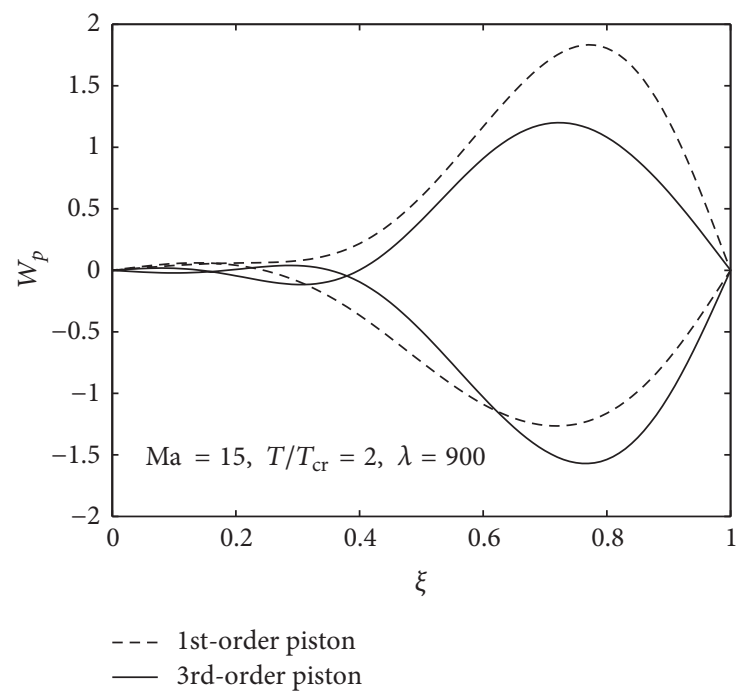

(c)

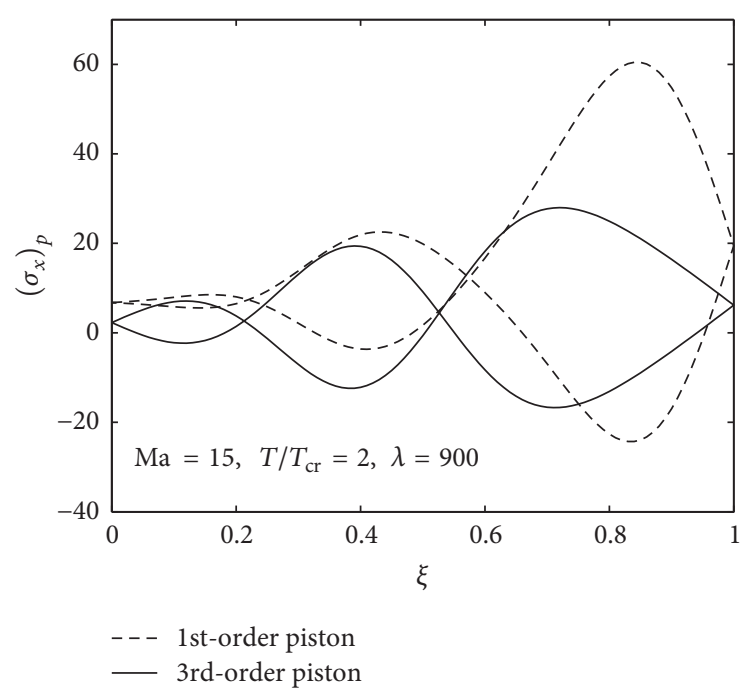

(e)

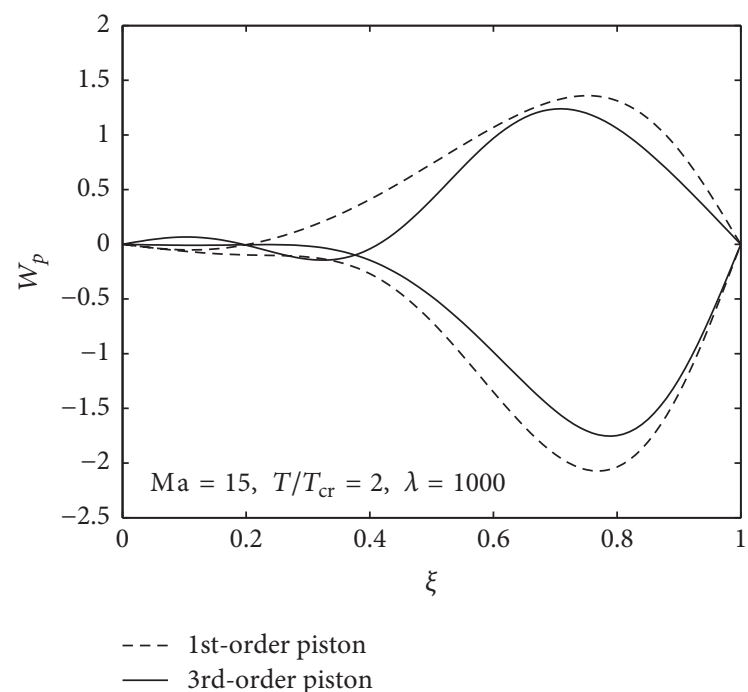

(d)

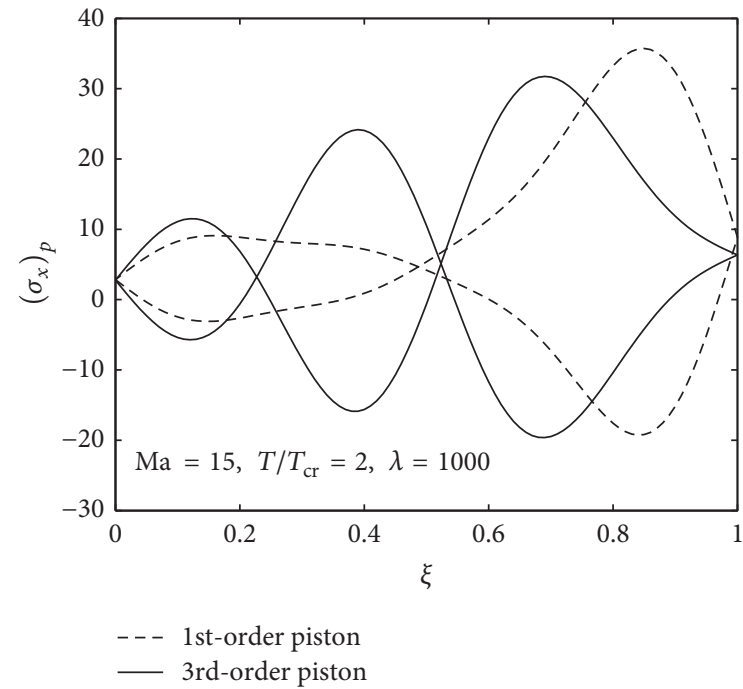

(f)

Figure 3: A square panel at $\mathrm{Ma}=15, T / T_{\mathrm{cr}}=2$ under different dynamic pressures: (a) phase portraits at $\lambda=900$; (b) phase portraits at $\lambda=1000$; (c) deflection shape in $x$ direction at $\lambda=900$; (d) deflection shape in $x$ direction at $\lambda=1000$; (e) stress distribution in $x$ direction at $\lambda=900$; (f) stress distribution in $x$ direction at $\lambda=1000$. 


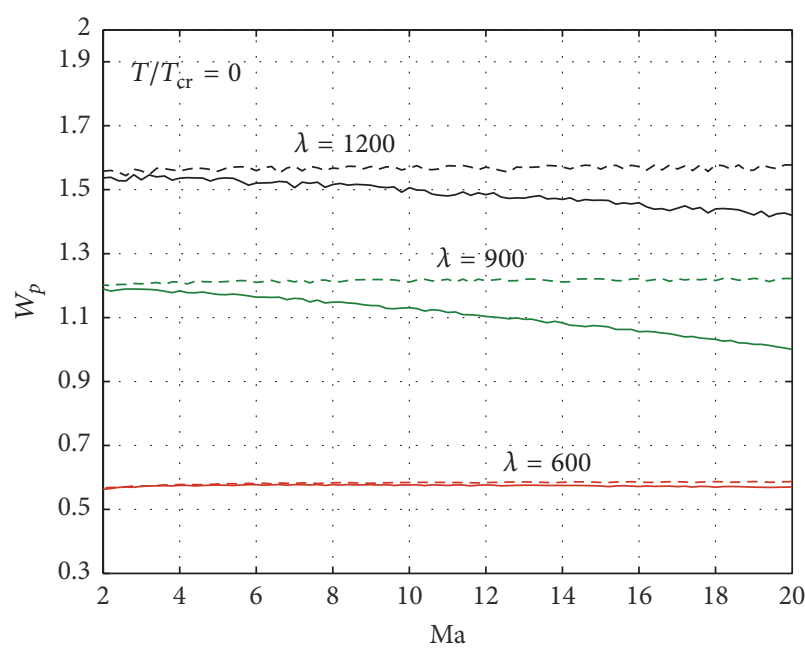

Dash line: first-order piston Solid line: third-order piston

(a)

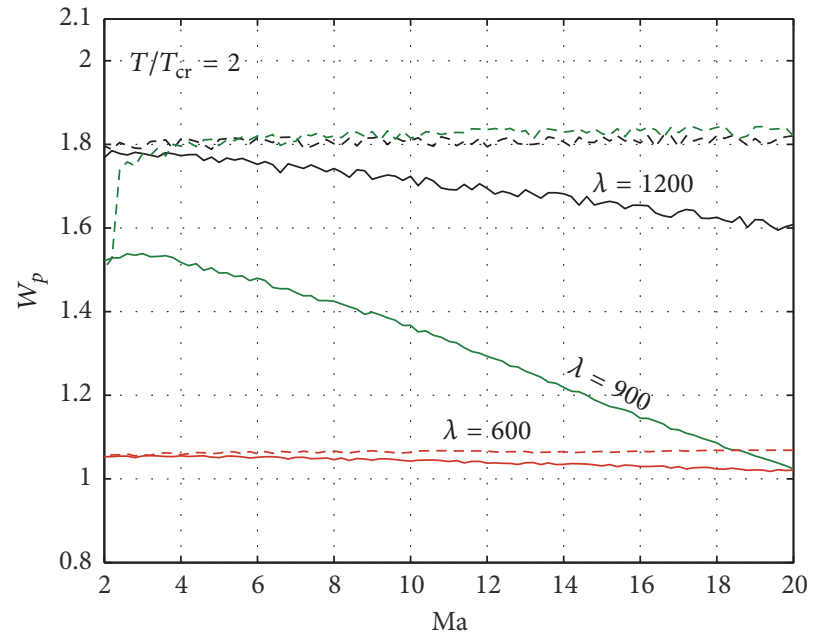

Dash line: first-order piston Solid line: third-order piston

Figure 4: Positive LCO deflection peak versus Mach number under several dynamic pressures for a square panel at different temperatures: (a) $T / T_{\text {cr }}=0$; (b) $T / T_{\text {cr }}=2$.

a quasi-periodic motion in contrast to the LCO periodic motion from the linear aerodynamics. In Figures 3(d) and 3(f), the deflection shapes and stress distributions are totally changed when the nonlinear aerodynamics is considered.

From Figure 2, we have shown that, for a given Mach number, there exists a critical dynamic pressure beyond which the deviation between the linear and nonlinear aerodynamic theories is large enough that the aerodynamic nonlinearity has to be considered. The temperature also has an influence on the critical dynamic pressure. Figures 4(a) and 4 (b) plot the curves in terms of $+W_{p}$ versus Ma for three dynamic pressures of $\lambda=600,900$, and 1200 at $T / T_{\text {cr }}=0$ and $T / T_{\text {cr }}=2$, respectively. Mach number is varied between $2<\mathrm{Ma}<20$. First, for a given $\lambda$, the deviation between the linear and nonlinear aerodynamics is augmented with the increase of Ma. Second, for a given $\mathrm{Ma}$, the deviation between the linear and nonlinear aerodynamics reaches a maximum at $\lambda=900$, which agrees with the conclusions from Figure 2. For a higher temperature at $T / T_{\mathrm{cr}}=2$, the same phenomena can be observed and the deviation between the linear and nonlinear aerodynamics is increased further. Therefore, for a given criterion value to evaluate the relative deviation between the linear and nonlinear aerodynamic theories, for example, $\epsilon=3 \%$, there is a critical dynamic pressure for a specific Mach number. The critical $\lambda_{\text {cr }}$ is defined as a dynamic pressure beyond which the linear and nonlinear aerodynamic theories produce different results with a relative deviation larger than $\epsilon$. For a larger Mach number, a lower $\lambda_{\mathrm{cr}}$ is obtained. With the increase of temperature, $\lambda_{\mathrm{cr}}$ is reduced further.

To sum up, the linear aerodynamic theory shows similar results for various Mach numbers, whereas, with the nonlinear aerodynamic theory, a larger Mach number produces a lower panel positive deflection peak. With the increase of dynamic pressure or temperature, the effect of the aerodynamic nonlinearity becomes more obvious. Particularly, for some specific dynamic pressures and temperatures, the nonlinear aerodynamics may produce different types of dynamic behaviors from the ones obtained using the linear aerodynamics. The deflection shape and stress distribution are changed accordingly, and the nonlinear aerodynamics has a larger influence on the stress distribution. By plotting the positive deflection peak $+W_{p}$ versus Ma under several dynamic pressures, for a given Mach number, there is a critical dynamic pressure $\lambda_{\text {cr }}$, beyond which the aerodynamic nonlinearity has to be considered.

\subsection{Observation of Bifurcation Diagrams with Linear and} Nonlinear Piston Theories. For the parameter sets with a larger dynamic pressure and a higher temperature, the panel may oscillate in a more complex pattern. The influence of aerodynamic nonlinearity on the evolution of complex behaviors is our crucial concern in the present study. Figure 5 shows the bifurcation diagrams at $\mathrm{Ma}=6, T / \mathrm{T}_{\mathrm{cr}}=6$ using the linear and nonlinear aerodynamics. The sweep in dynamic pressure is done from $\lambda=1200$ to $\lambda=100$ with a step increment of $\Delta \lambda=-5$. With the increase of dynamic pressure, an evolution from a buckling to chaos and then to a LCO can be observed in both Figures 5(a) and 5(b), which is similar to the results of an airfoil model studied by Dai et al. [30]. Between $1000<\lambda<1200$, a period-1 motion is obtained with the linear aerodynamics, while the nonlinear aerodynamic loading produces a period- 2 motion. Generally speaking, the aerodynamic nonlinearity does not influence the bifurcation diagram very much at $\mathrm{Ma}=6, T / T_{\mathrm{cr}}=6$.

Nevertheless, what may happen for a larger Mach number? First, we increase the Mach number up to $\mathrm{Ma}=10$ and even $\mathrm{Ma}=15$ with the temperature the same as $T / T_{\mathrm{cr}}=6$. 


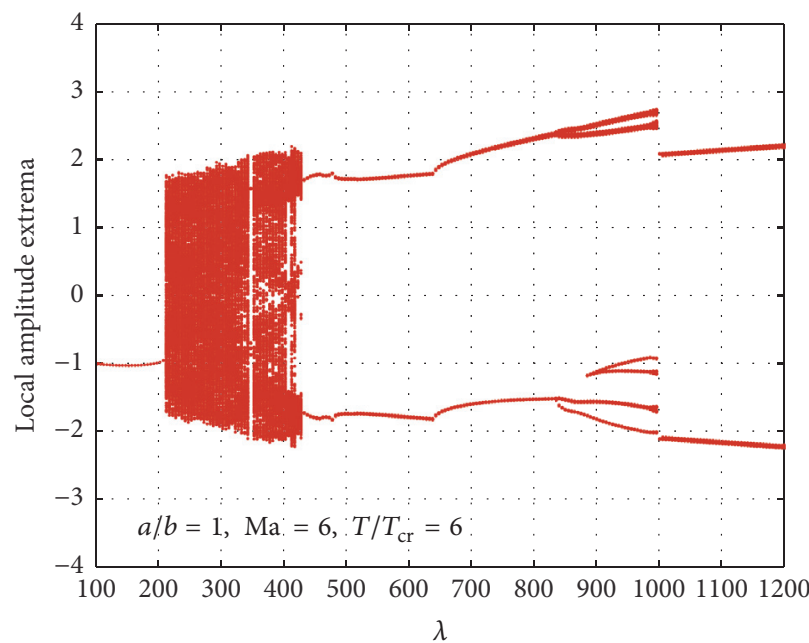

- 1st-order piston

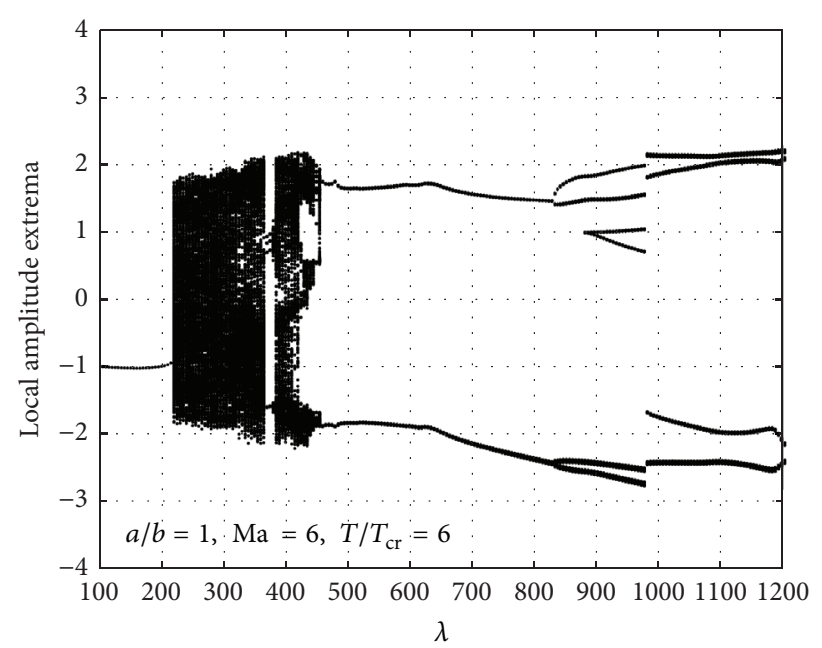

- 3rd-order piston

(a)

(b)

Figure 5: Bifurcation diagrams for a square panel at $\mathrm{Ma}=6, \mathrm{~T} / \mathrm{T}_{\mathrm{cr}}=6$ : (a) first-order piston theory; (b) third-order piston theory.

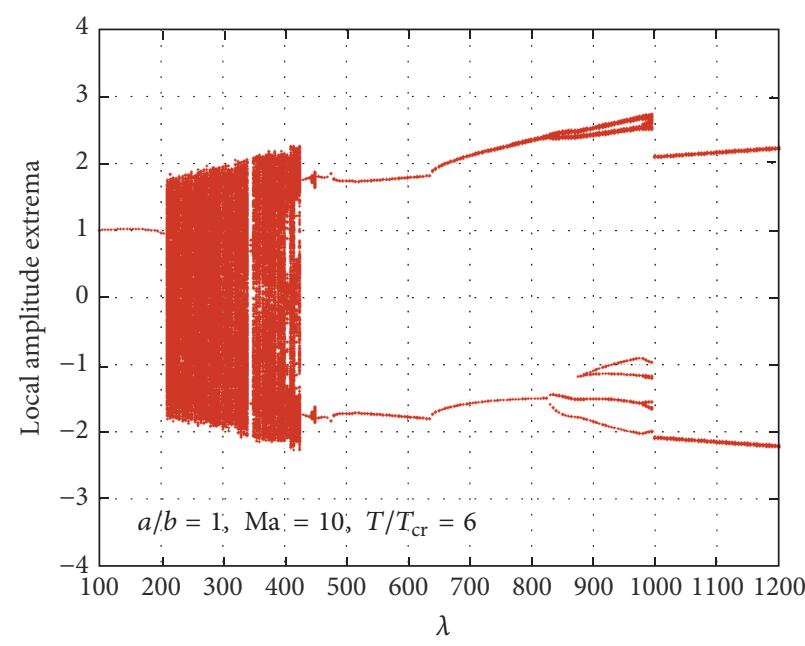

- 1st-order piston

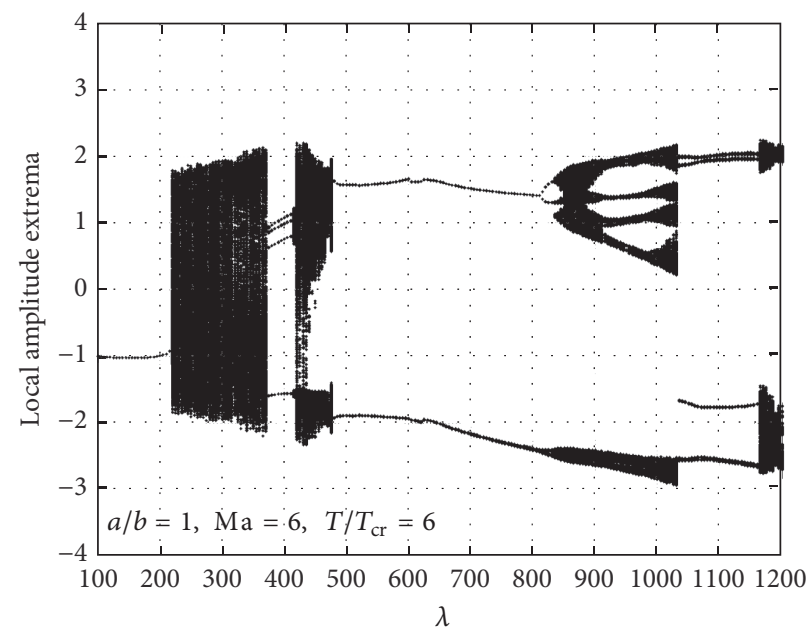

- 3rd-order piston

(a)

FIgURE 6: Bifurcation diagrams for a square panel at $\mathrm{Ma}=10, T / T_{\mathrm{cr}}=6:$ (a) first-order piston theory; (b) third-order piston theory.

Using the linear and nonlinear aerodynamics the resultant bifurcation diagrams in terms of local deflection extrema versus dynamic pressure are shown in Figure 6 for $\mathrm{Ma}=$ $10, T / T_{\mathrm{cr}}=6$. It is obvious that the bifurcation diagram in Figure 6(a) is almost the same as the one in Figure 5(a). This agrees with the conclusion aforementioned that the linear aerodynamic loading produces similar results for various Mach number. Compared to Figure 6(a), the nonlinear aerodynamic loading obtains a very different bifurcation diagram as shown in Figure 6(b). First, the bifurcation diagram in Figure 6(a) shows that, with the increase of dynamic pressure, the panel oscillates as LCO when $\lambda>450$. In contrast, the panel undergoes some chaotic and nonsimple periodic oscillations as shown in Figure 6(b). Specifically, Figure 7 shows the phase portraits and Poincaré maps for several dynamic pressures in Figure 6. The results show that the linear aerodynamic loading results in period-1, period-2, and period-4 motions, while the nonlinear aerodynamic loading produces mainly chaotic motions except the period-2 and multiperiod motions for $\lambda=1100$ and $\lambda=1200$, respectively.

For a higher $\mathrm{Ma}=15$, Figure 8 (a) shows that the linear aerodynamic loading still obtains the similar bifurcation diagram to that for $\mathrm{Ma}=6$ and $\mathrm{Ma}=10$. However, the aerodynamic nonlinearity results in a more complex bifurcation diagram as shown in Figure 8(b). First, the chaos region from the nonlinear aerodynamics $(220<\lambda<455)$ is 


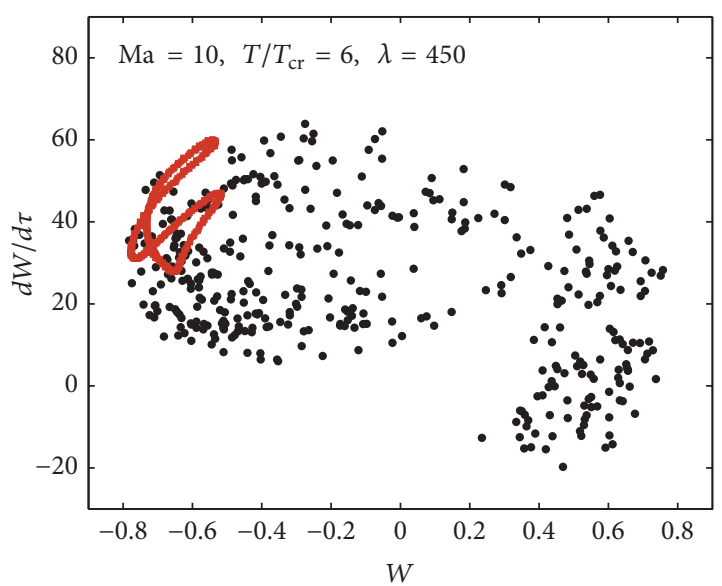

- 3rd-order piston

- 1st-order piston

(a)

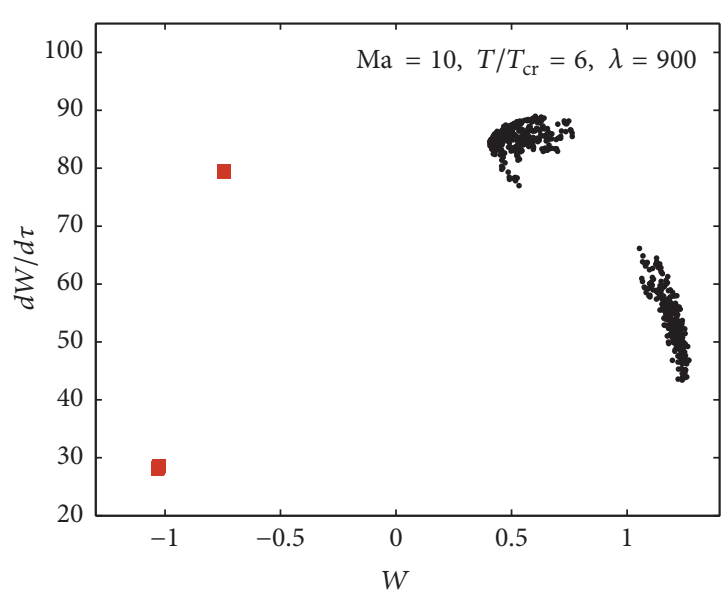

- 1st-order piston

- 3rd-order piston

(c)

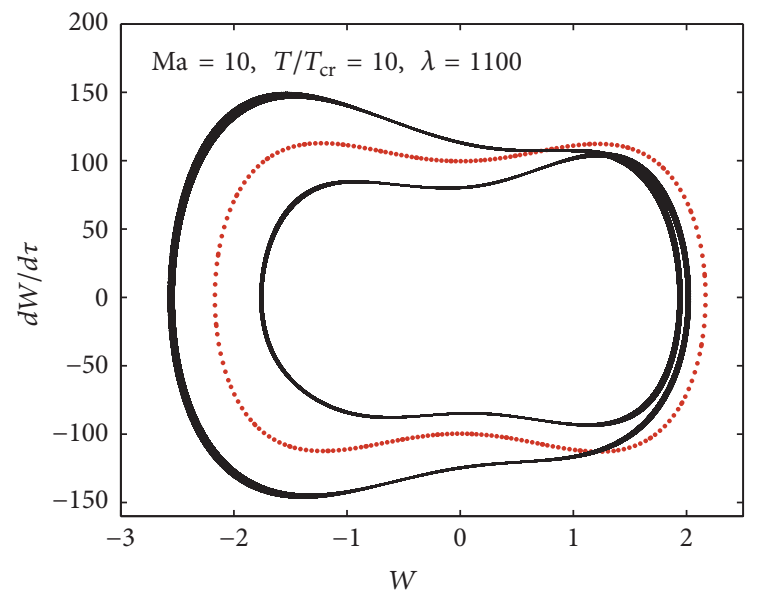

- 1st-order piston

- 3rd-order piston

(e)

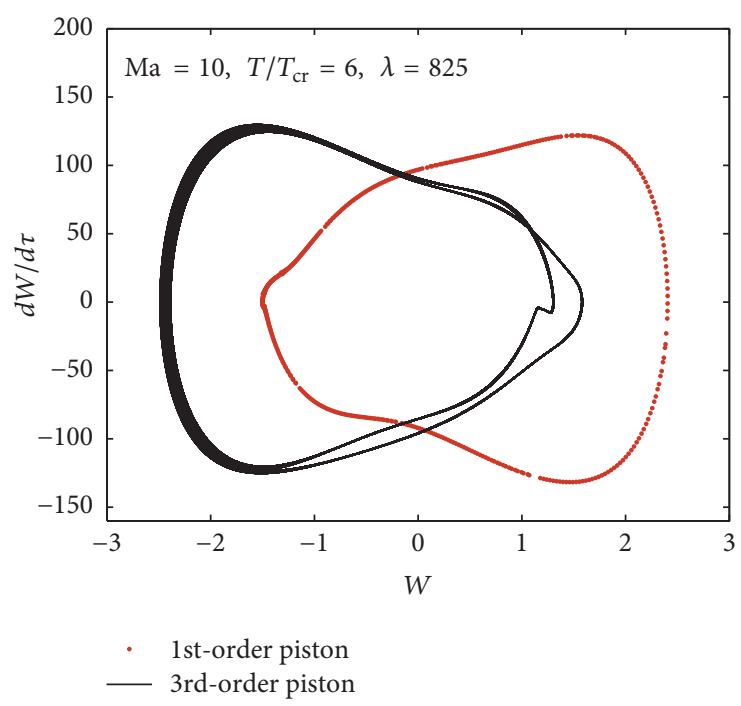

(b)

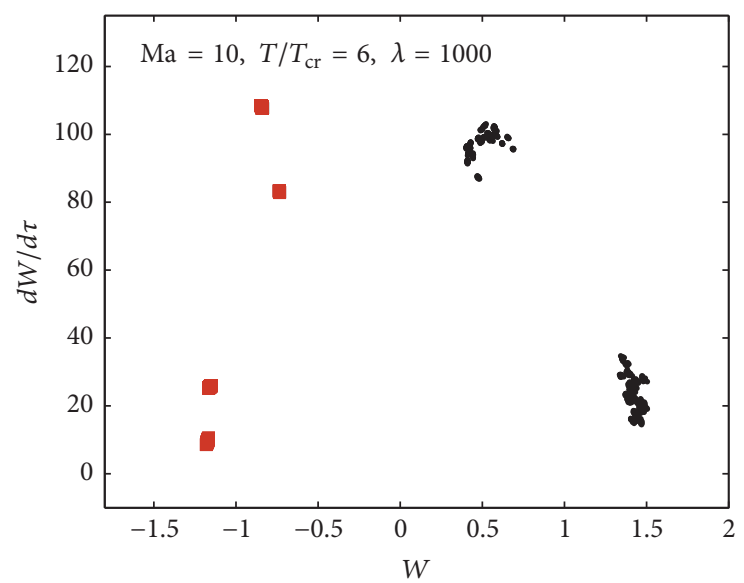

- 1st-order piston

- 3rd-order piston

(d)

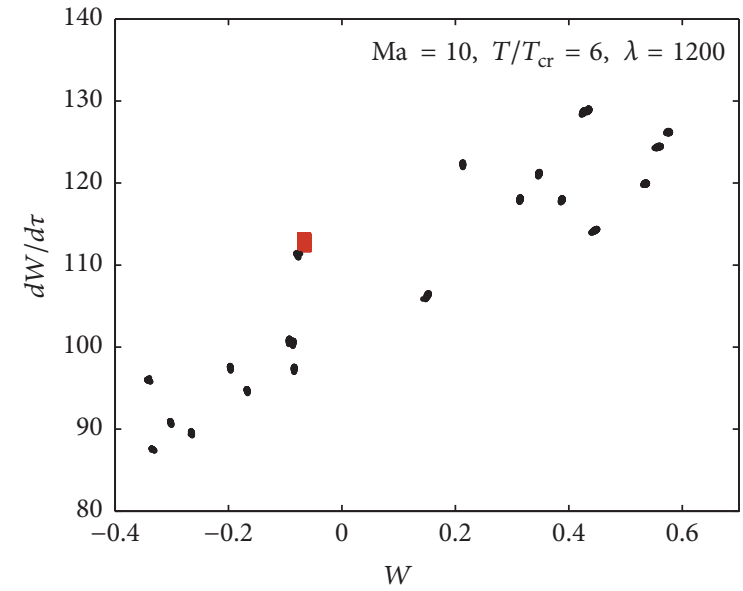

- 1st-order piston

- 3rd-order piston

(f)

Figure 7: A square panel at $\mathrm{Ma}=10, T / T_{\mathrm{cr}}=6$ under different dynamic pressures: (a) Poincaré map at $\lambda=450$; (b) phase portrait at $\lambda=825$; (c) Poincaré map at $\lambda=900$; (d) Poincaré map at $\lambda=1000$; (e) phase portrait at $\lambda=1100$; (f) Poincaré map at $\lambda=1200$. 


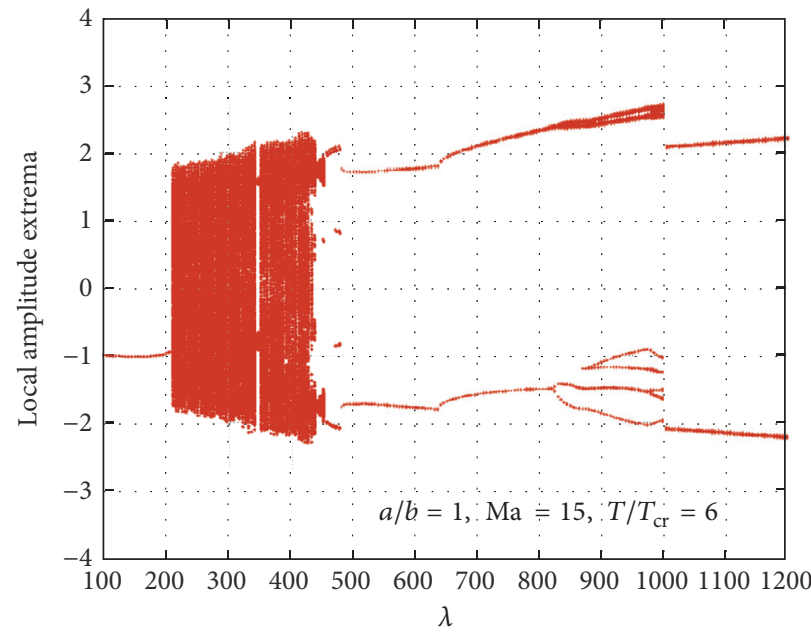

- 1st-order piston

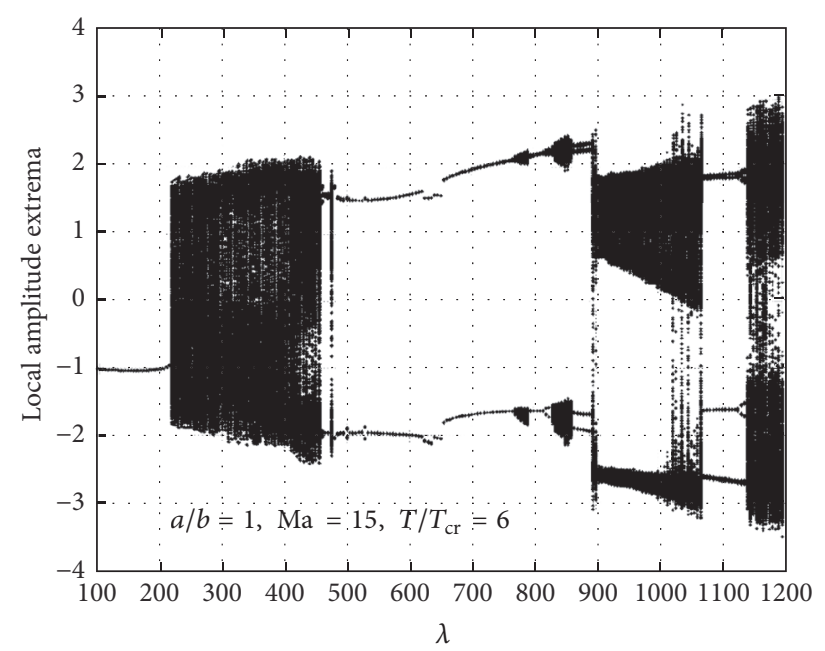

- 3rd-order piston

(a)

(b)

FIgURE 8: Bifurcation diagrams for a square panel at $\mathrm{Ma}=15, T / T_{\mathrm{cr}}=6$ : (a) first-order piston theory; (b) third-order piston theory.

broader than that from the linear aerodynamics $(210<\lambda<$ 435). For $435<\lambda<455$ a quasi-periodic motion is observed with the linear aerodynamics. Second, between $480<\lambda<$ 760 , LCO motions are obtained from both the linear and nonlinear aerodynamics. The nonlinear aerodynamic loading produces a smaller $+W_{p}$ and larger $\left|-W_{p}\right|$ than those from the linear aerodynamics. This is consistent with the conclusion in [14] that the nonlinear aerodynamic loading acts with an overpressure to push the panel into the cavity. Third, the bifurcation diagrams are totally different between $760<\lambda<1200$, for which the linear aerodynamic loading results in periodic motions including period-1 and multiperiod motions. By contrast, the nonlinear aerodynamic loading almost produces all chaotic motions except for the period-1 motions between $1080<\lambda<1140$.

To make the comparison in Figures 8 (a) and 8 (b) easily visible, several dynamic pressures are considered further as shown in Figure 9. For $\lambda=600$, Figure 9(a) presents the period-1 motions from both the linear and nonlinear aerodynamics but with various $+W_{p}$ and $-W_{p}$. For $\lambda=$ 850 in Figure 9(b), a period-2 and quasi-periodic motions are observed with the linear and nonlinear aerodynamics, respectively. Similarly, Figure 9(c) shows a period-1 and period-2 motions for $\lambda=1110$ from the linear and nonlinear aerodynamics, respectively. Figures $9(\mathrm{~d})$ and $9(\mathrm{e})$ show the Poincaré maps for $\lambda=1000$ and $\lambda=1200$, and the chaotic motions are observed with the nonlinear aerodynamics; by contrast, period- 4 and period- 1 motions are obtained with the linear aerodynamics. Obviously, the evolution of dynamic behaviors considering the aerodynamic nonlinearity is much more complex, especially for a larger Mach number under a larger dynamic pressure. The complex behaviors from the nonlinear aerodynamics are due to the interplay between the nonlinear membrane stresses and nonlinear aerodynamics, that is, the interplay between the "hard spring" and "soft spring" aforementioned. For panel flutter designs, the prediction of panel fatigue life is a crucial concern. The algorithms for predicting fatigue life for LCO, nonsimple harmonic, and quasi-periodic versus chaotic motions are different; thus, the nonlinear aerodynamic theory may have to be employed for a reliable fatigue life prediction.

\subsection{Effects of Each Nonlinear Aerodynamic Term in Third-} Order Piston Theory. The numerical simulations above are all based on the full third-order piston theory accounting for the nonlinear aerodynamics. Of course, the additional nonlinear aerodynamic terms in (11) increase the computational costs compared to the linear piston theory. Hence, in this section we aim to evaluate the effects of each nonlinear term in the full third-order piston theory and then retain the most significant one and neglect the others. In detail, we switch on/off the terms of $C_{2 t}, C_{2 x}, C_{3 t}$, and $C_{3 x}$ sequentially and plot the curves in terms of $+W_{p}$ versus $\lambda$ at $\mathrm{Ma}=20, T / T_{\mathrm{cr}}=0$.

Figure 10 presents the curves aforementioned comparing with those from the linear piston theory $\left(C_{1 t}=C_{1 x}=1\right)$. Particularly, Figure 10(a) shows the results by an inclusion of each second-order term. The curve denoted by $C_{2 t}=1$ is consistent with the curve denoted by the first-order piston theory, while the curves denoted by $C_{2 x}=1$ and $C_{2 t}=$ $C_{2 x}=1$ are consistent with the curve denoted by the thirdorder piston theory. Hence, the term of $(\partial w / \partial x)^{2}$ is proved to be the most significant in the full third-order piston theory. Similarly, Figure 10(b) shows the results by an inclusion of each third-order term, the curves denoted by which are all consistent with the curve denoted by the first-order piston theory. Therefore, we can come to a conclusion that each third-order nonlinear term has little influence on the full third-order piston theory. 


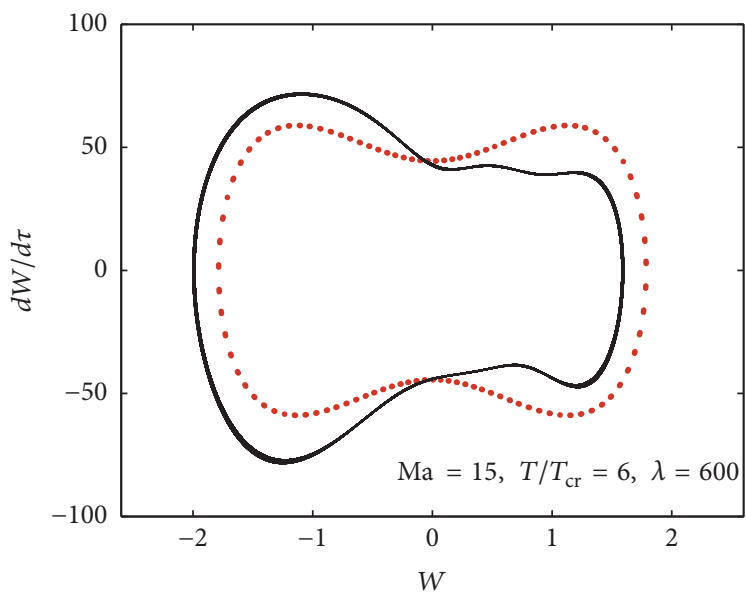

- 1st-order piston — 3rd-order piston

(a)

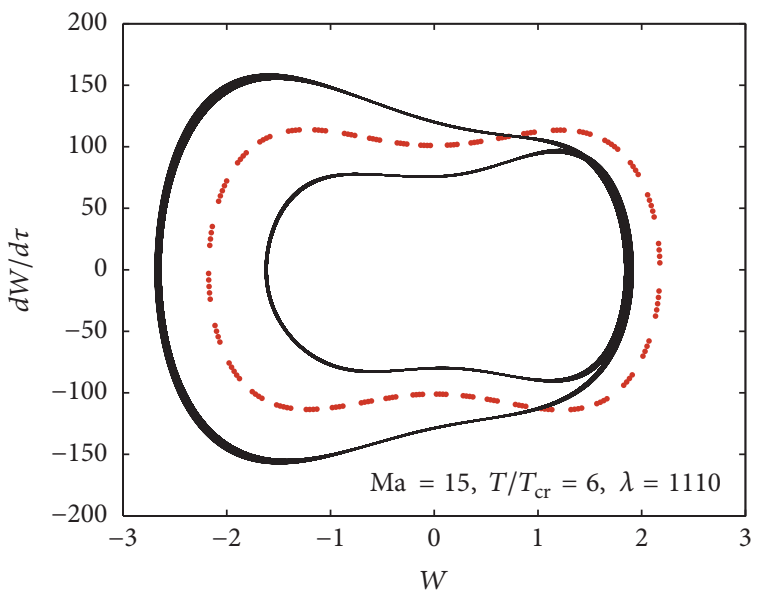

- 1st-order piston — 3rd-order piston

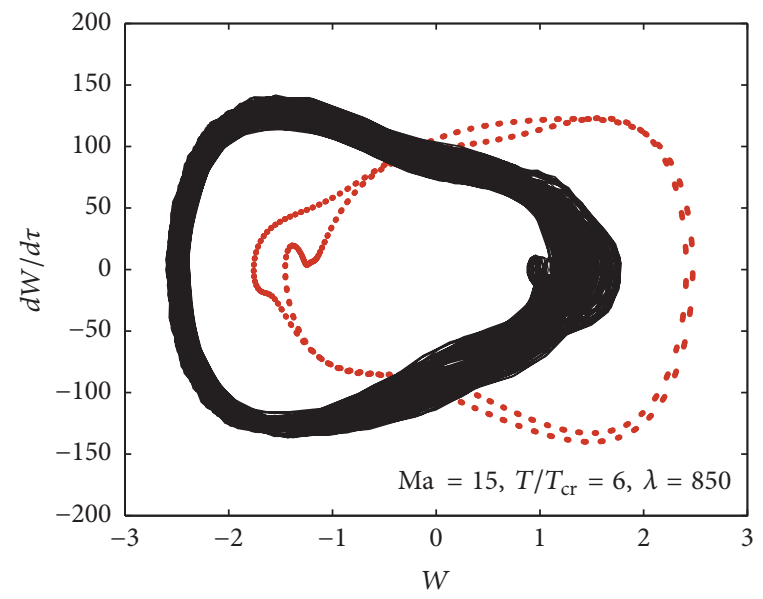

- 1st-order piston

— 3rd-order piston

(b)

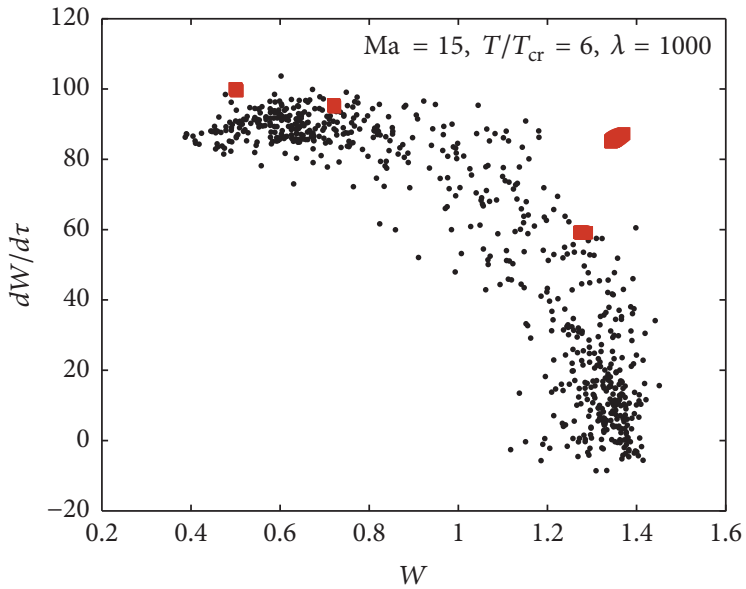

- 3rd-order piston

- 1st-order piston

(c)

(d)

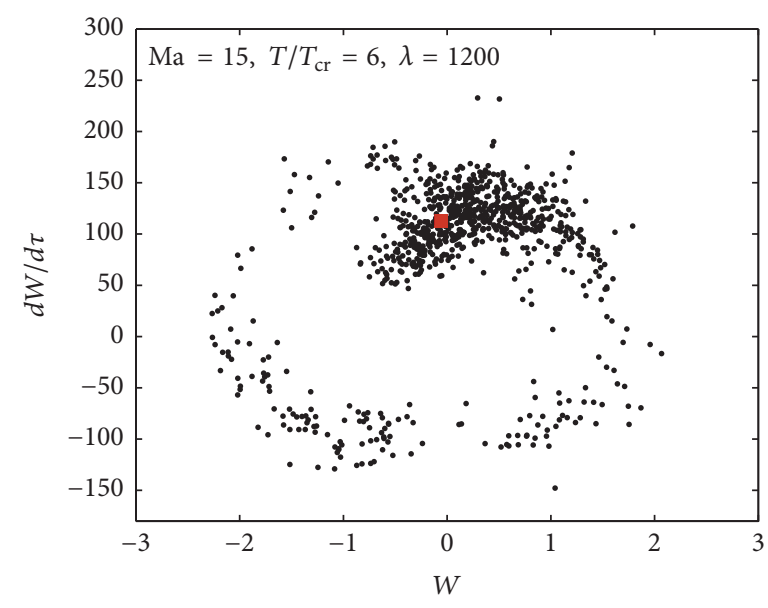

- 3rd-order piston

- 1st-order piston

(e)

Figure 9: A square panel at $\mathrm{Ma}=15, T / T_{\mathrm{cr}}=6$ under different dynamic pressures: (a) phase portraits at $\lambda=600$; (b) phase portraits at $\lambda=850$; (c) phase portraits at $\lambda=1110$; (d) Poincaré maps at $\lambda=1000$; (e) Poincaré maps at $\lambda=1200$. 
TABLE 1: Discussion issues for a fluttering panel: comparisons with literature results.

\begin{tabular}{lcccc}
\hline Discussion issues & Eastep and McIntosh Jr. (1971) & Gray et al. (1991) & Cheng and Mei (2004) & Present work \\
\hline Nonlinear term effects & $\sqrt{ }$ & $\sqrt{ }$ & $\backslash$ & $\sqrt{ }$ \\
LCO deflection peak & $\sqrt{ }$ & $\sqrt{ }$ & $\sqrt{ }$ \\
Bifurcation diagram & $\backslash$ & $\backslash$ & $\backslash$ & $\sqrt{ }$ \\
Effects of Ma, $\lambda, T / T_{\mathrm{cr}}$ & $\backslash$ & $\backslash$ & $\sqrt{ }$ \\
\hline
\end{tabular}

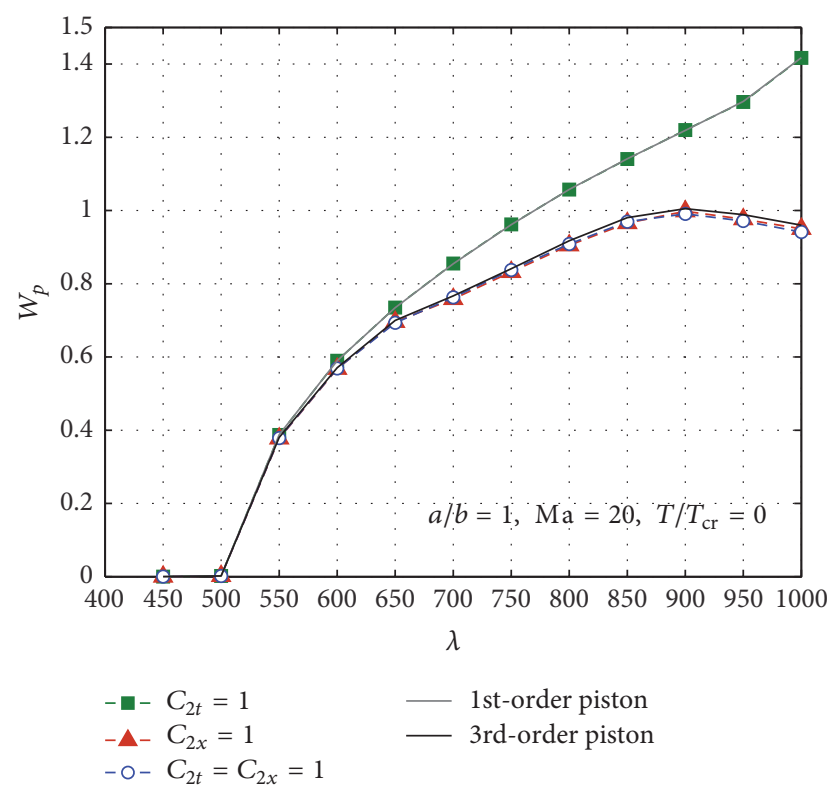

(a)

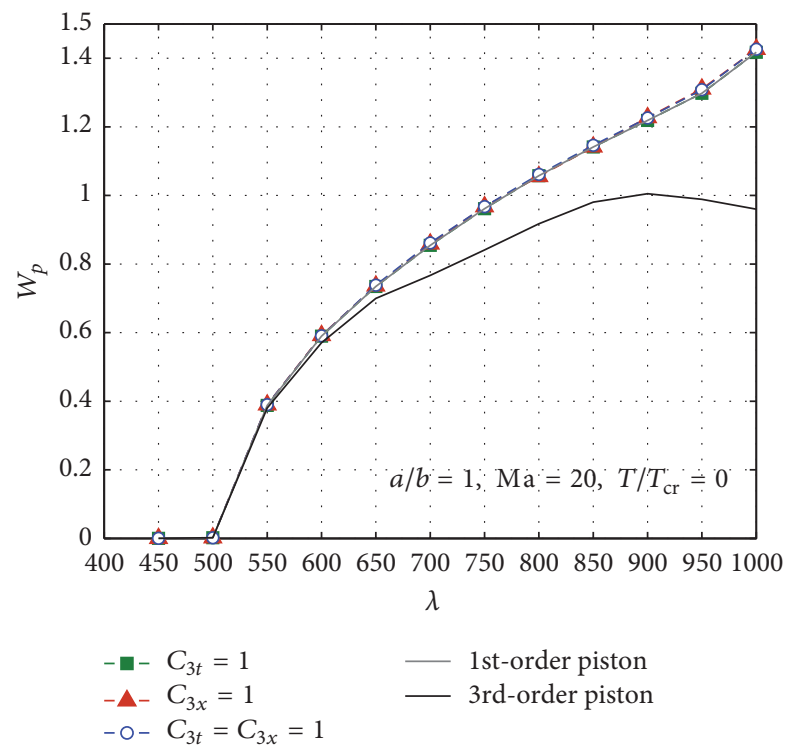

(b)

FIGURE 10: The effects of each nonlinear aerodynamic term: positive LCO deflection peak versus dynamic pressure for a square panel at Ma $=20, T / T_{\text {cr }}=0$ : (a) each term of second-order piston theory; (b) each term of third-order piston theory.

Based on the influence of each nonlinear aerodynamic term, for the panels studied in the present paper, the thirdorder piston theory can be simplified as

$$
p-p_{\infty}=\frac{2 q}{\beta}\left[\frac{1}{U} \frac{\partial w}{\partial t}+\frac{\partial w}{\partial x}+\frac{\gamma+1}{4} \mathrm{Ma}\left(\frac{\partial w}{\partial x}\right)^{2}\right] .
$$

Using the simplified third-order piston theory, that is, the linear piston theory added to the second-order term of $(\partial w / \partial x)^{2}$, the derivation of the nonlinear aeroelastic equations will be much simpler and consequently the computational time for solving the ODEs will be reduced.

3.4. Comparisons with Literature Results. Based on the discussions above in terms of the effects of nonlinear aerodynamics to the LCO deflection, to the bifurcation diagrams and effects of each nonlinear aerodynamic term, here a comparison between the available literature results and the present work is presented. We mainly provide the discussion issues for each research work, which is shown in Table 1. The comparison results show that the present work provides extended discussions about the effects of nonlinear aerodynamics to the panel flutter in hypersonic flow. Through the current work, the nonlinear aerodynamics produce different bifurcation boundaries, which will influence the panel fatigue life prediction. In addition, the combination of parameters $\mathrm{Ma}, \lambda$, and $T / T_{\mathrm{cr}}$ interacts with nonlinear aerodynamics, which has not been discussed in the literatures available.

\section{Concluding Remarks}

The present study evaluates the aerodynamic nonlinearity by comparing the third-order piston theory with the first-order piston theory. A simply supported square plate fluttering at hypersonic speeds is studied considering both the airflow and structural nonlinearities. The third-order piston theory and von Karman plate theory are used accounting for nonlinear aerodynamic loading and nonlinear strain-displacement relation, respectively. The Galerkin method and numerical RK4 are applied to obtain the ODEs and perform the time integration. LCO deflection and bifurcation diagrams versus dynamic pressure are both observed with the first-order and third-order piston theories. The influences of Mach number, dynamic pressure, and temperature on the aerodynamic nonlinearity are evaluated, and they all affect the choice of the nonlinear aerodynamic theory. The following conclusions can be drawn from the numerical results:

(1) With the first-order piston theory, the results for various Mach number are almost the same LCO 
motions. By contrast, the third-order piston theory produces the LCO motion with a smaller positive deflection peak for a higher Mach number.

(2) With the increase of Mach number, the deviation between the first-order and third-order piston theories is increased. The same phenomena can be observed with the increase of dynamic pressure and temperature.

(3) For a specific Mach number, there is a critical dynamic pressure $\lambda_{\mathrm{cr}}$, beyond which the nonlinear aerodynamic theory has to be used. A smaller $\lambda_{\text {cr }}$ is obtained for a higher temperature.

(4) A LCO motion obtained with the linear aerodynamic theory may turn out to be a quasi-periodic or chaotic motion when the aerodynamic nonlinearity is considered. And, the third-order piston theory can be simply represented by adding the nonlinear term $(\partial w / \partial x)^{2}$ of the third-piston theory to the linear piston theory.

\section{Nomenclature}

$\begin{array}{ll}a, b: & \text { Plate length, plate width, } \mathrm{m} \\ D: & \text { Plate stiffness, } \mathrm{Nm} \\ E: & \text { Young's modulus, } \mathrm{N} / \mathrm{m}^{2} \\ h: & \text { Plate thickness, } \mathrm{m} \\ M: & \text { Number of modes in Galerkin method } \\ \mathrm{Ma}: & \text { Mach number } \\ m, n: & \text { Harmonic mode number } \\ N_{x}^{T}, N_{y}^{T}: & \text { In-plane thermal force in } x, y \text { directions, } \mathrm{N} / \mathrm{m} \\ p-p_{\infty}: & \text { Aerodynamic pressure, } \mathrm{N} / \mathrm{m}^{2} \\ q: & \rho U^{2} / 2, \text { dynamic pressure }, \mathrm{N} / \mathrm{m}^{2} \\ R_{x}^{T}, R_{y}^{T}: & \text { Nondimensional in-plane thermal loads } \\ r, s: & \text { Harmonic mode number } \\ T: & \text { Temperature differential, } \mathrm{K} \\ t: & \text { Time, } \mathrm{s} \\ U: & \text { Velocity, } \mathrm{m} / \mathrm{s} \\ w: & \text { Panel transverse deflection, } \mathrm{m} \\ x, y: & \text { Streamwise, spanwise coordinate, } \mathrm{m} \\ \alpha: & \text { Thermal expansion coefficient, } /{ }^{\circ} \mathrm{C} \\ \beta: & \left.\text { (Ma }{ }^{2}-1\right)^{1 / 2} \\ \nu: & \text { Poisson ratio } \\ \rho, \rho_{m}: & \text { Air density, plate density, } \mathrm{kg} / \mathrm{m}^{3} \\ \Phi: & \text { Airy stress function. } \\ & \end{array}$

Subscripts

$p$ : Peak.

\section{Conflicts of Interest}

The authors declare that they have no conflicts of interest.

\section{References}

[1] E. H. Dowell, "Nonlinear oscillations of a fluttering plate," AIAA Journal, vol. 4, no. 7, pp. 1267-1275, 1966.

[2] Z. Jian, Y. Zhichun, and G. Yingsong, "Aeroelastic stability analysis of heated panel with aerodynamic loading on both surfaces," Science China Technological Sciences, vol. 55, no. 10, pp. 2720-2726, 2012.

[3] D. Xie, M. Xu, and E. H. Dowell, "Proper orthogonal decomposition reduced-order model for nonlinear aeroelastic oscillations," AIAA Journal, vol. 52, no. 2, pp. 229-241, 2014.

[4] D. Xie, M. Xu, and E. H. Dowell, "Projection-free proper orthogonal decomposition method for a cantilever plate in supersonic flow," Journal of Sound and Vibration, vol. 333, no. 23, pp. 6190-6208, 2014.

[5] D. Xie and M. Xu, "A comparison of numerical and semianalytical proper orthogonal decomposition methods for a fluttering plate," Nonlinear Dynamics, vol. 79, no. 3, pp. 19711989, 2015.

[6] E. H. Dowell, "Nonlinear oscillations of a fluttering plate. II.," AIAA Journal, vol. 5, no. 10, pp. 1856-1862, 1967.

[7] M. Berci, "Lift-deficiency functions of elliptical wings in incompressible potential flow: Jones' theory revisited," Journal of Aircraft, vol. 53, no. 2, pp. 599-602, 2016.

[8] M. F. El-Sayed, N. T. Eldabe, M. H. Haroun, and D. M. Mostafa, "Nonlinear electroviscoelastic potential flow instability theory of two superposed streaming dielectric fluids," Canadian Journal of Physics, vol. 92, no. 10, pp. 1249-1257, 2014.

[9] E. Carrera, E. Zappino, G. Augello, A. Ferrarese, and M. Montabone, "Panel flutter analysis of curved panels for launchers applications," in Proceedings of the 7th European Symposium on Aerothermodynamics for Space Vehicles, Paris, France, 2011.

[10] E. Carrera and E. Zappino, "Aeroelastic analysis of pinched panels in supersonic flow changing with altitude," Journal of Spacecraft and Rockets, vol. 51, no. 1, pp. 187-199, 2014.

[11] E. Carrera, E. Zappino, K. Patočka et al., "Aeroelastic analysis of versatile thermal insulation (VTI) panels with pinched boundary conditions," CEAS Space Journal, vol. 6, no. 1, pp. 2335, 2014.

[12] G. Mei, J. Zhang, G. Xi, X. Sun, and J. Chen, "Analysis of supersonic and transonic panel flutter using a fluid-structure coupling algorithm," Journal of Vibration and Acoustics, vol. 136, no. 3, Article ID 031013, 2014.

[13] K.-N. Koo and W.-S. Hwang, "Effects of hysteretic and aerodynamic damping on supersonic panel flutter of composite plates," Journal of Sound and Vibration, vol. 273, no. 3, pp. 569-583, 2004.

[14] S. C. McIntosh Jr. and J. I. Lerner, "Theoretical considerations of some nonlinear aspects of hypersonic panel flutter," Final Report, 1965.

[15] F. E. Eastep and S. C. McIntosh Jr., "Analysis of nonlinear panel flutter and response under random excitation or nonlinear aerodynamic loading," AIAA Journal, vol. 9, no. 3, pp. 411-418, 1971.

[16] S. C. McIntosh Jr., "Effect of hypersonic nonlinear aerodynamic loading on panel flutter," AIAA Journal, vol. 11, no. 1, pp. 29-32, 1973.

[17] M. Goland, "An exact solution for two-dimensional linear panel flutter at supersonic speeds," Journal of the Aeronautical Sciences, vol. 21, no. 4, pp. 275-276, 2012.

[18] C. E. Gray, C. Mei, and C. P. Shore, "Finite element method for large-amplitude two-dimensional panel flutter at hypersonic speeds," AIAA Journal, vol. 29, no. 2, pp. 290-298, 1991.

[19] G. Cheng and C. Mei, "Finite element modal formulation for hypersonic panel flutter analysis with thermal effects," AIAA Journal, vol. 42, no. 4, pp. 687-695, 2004. 
[20] L. X. Peng, "Free vibration analysis of symmetrically laminated folded plate structures using an element-free Galerkin method," Mathematical Problems in Engineering, vol. 2015, Article ID 124296, 13 pages, 2015.

[21] A. Sarkar and M. P. Païdoussis, "A cantilever conveying fluid: coherent modes versus beam modes," International Journal of Non-Linear Mechanics, vol. 39, no. 3, pp. 467-481, 2004.

[22] L.-K. Yao, B. He, Y. Zhang, and W. Zhou, "Semi-analytical finite strip transfer matrix method for buckling analysis of rectangular thin plates," Mathematical Problems in Engineering, vol. 2015, Article ID 485686, 11 pages, 2015.

[23] W. Sun, Y. Liu, and G. Du, "Analytical modeling of hard-coating cantilever composite plate considering the material nonlinearity of hard coating," Mathematical Problems in Engineering, vol. 2015, Article ID 978392, 14 pages, 2015.

[24] H.-H. Dai, J. K. Paik, and S. N. Atluri, "The global nonlinear galerkin method for the analysis of elastic large deflections of plates under combined loads: a scalar homotopy method for the direct solution of nonlinear algebraic equations," Computers, Materials and Continua, vol. 23, no. 1, pp. 69-99, 2011.

[25] C. Gang, S. Jian, and L. Yueming, "Active flutter suppression control law design method based on balanced proper orthogonal decomposition reduced order model," Nonlinear Dynamics, vol. 70, no. 1, pp. 1-12, 2012.

[26] G. Giunta and S. Belouettar, "Higher-order hierarchical models for the free vibration analysis of thin-walled beams," Mathematical Problems in Engineering, vol. 2015, Article ID 940347, 12 pages, 2015.

[27] B. I. Epureanu, L. S. Tang, and M. P. Païdoussis, "Coherent structures and their influence on the dynamics of aeroelastic panels," International Journal of Non-Linear Mechanics, vol. 39, no. 6, pp. 977-991, 2004.

[28] M. Amabili, A. Sarkar, and M. P. Païdoussis, "Reduced-order models for nonlinear vibrations of cylindrical shells via the proper orthogonal decomposition method," Journal of Fluids and Structures, vol. 18, no. 2, pp. 227-250, 2003.

[29] D. Xie, M. Xu, H. Dai, and E. H. Dowell, "Proper orthogonal decomposition method for analysis of nonlinear panel flutter with thermal effects in supersonic flow," Journal of Sound and Vibration, vol. 337, pp. 263-283, 2015.

[30] H. Dai, X. Yue, J. Yuan, D. Xie, and S. N. Atluri, "A comparison of classical Runge-Kutta and Henon's methods for capturing chaos and chaotic transients in an aeroelastic system with freeplay nonlinearity," Nonlinear Dynamics, vol. 81, no. 1-2, pp. 169-188, 2015.

[31] H. Dai, X. Wang, M. Schnoor, and S. N. Atluri, "Analysis of internal resonance in a two-degree-of-freedom nonlinear dynamical system," Communications in Nonlinear Science and Numerical Simulation, vol. 49, pp. 176-191, 2017. 


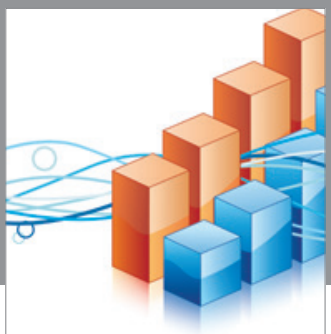

Advances in

Operations Research

vatem alat4

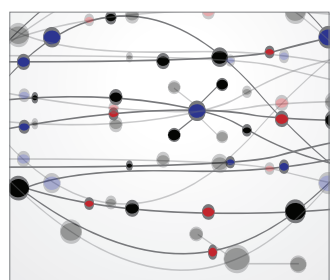

\section{The Scientific} World Journal
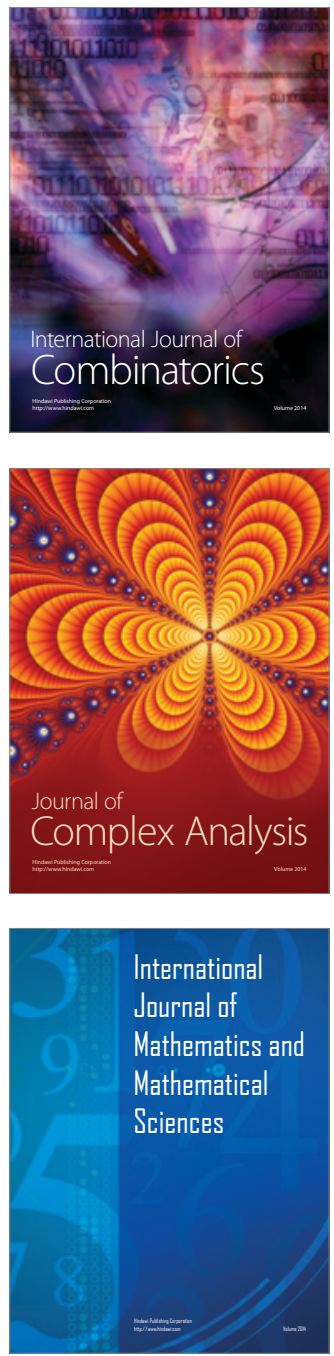
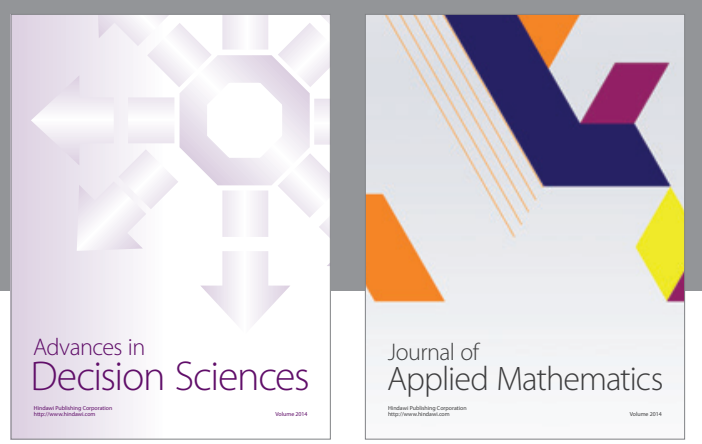

Algebra

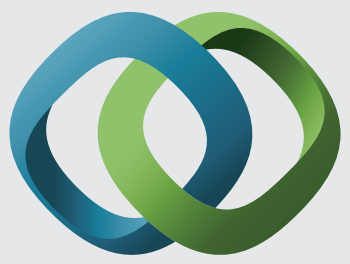

\section{Hindawi}

Submit your manuscripts at

https://www.hindawi.com
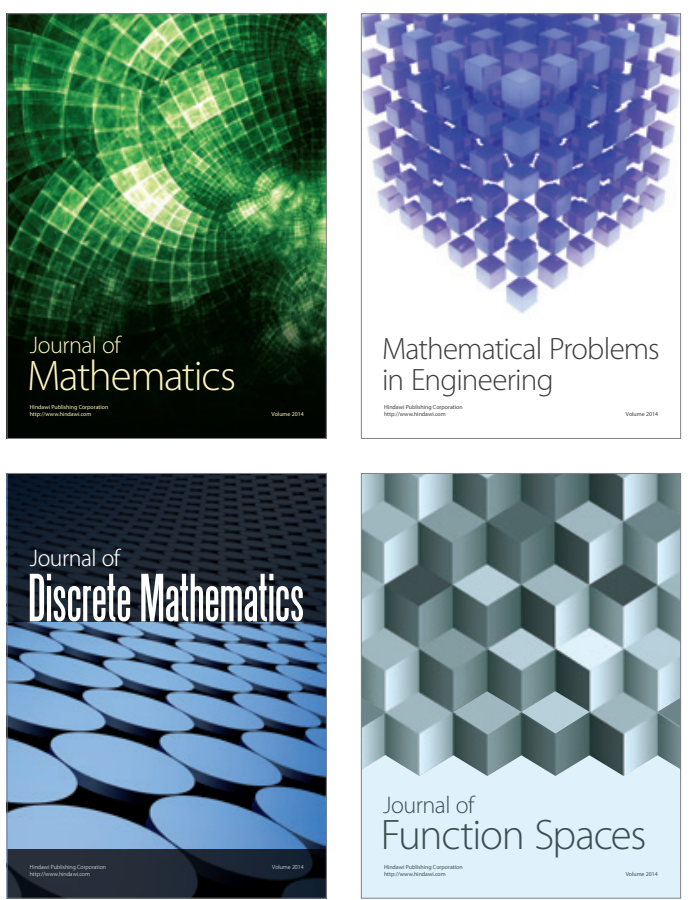

Mathematical Problems in Engineering
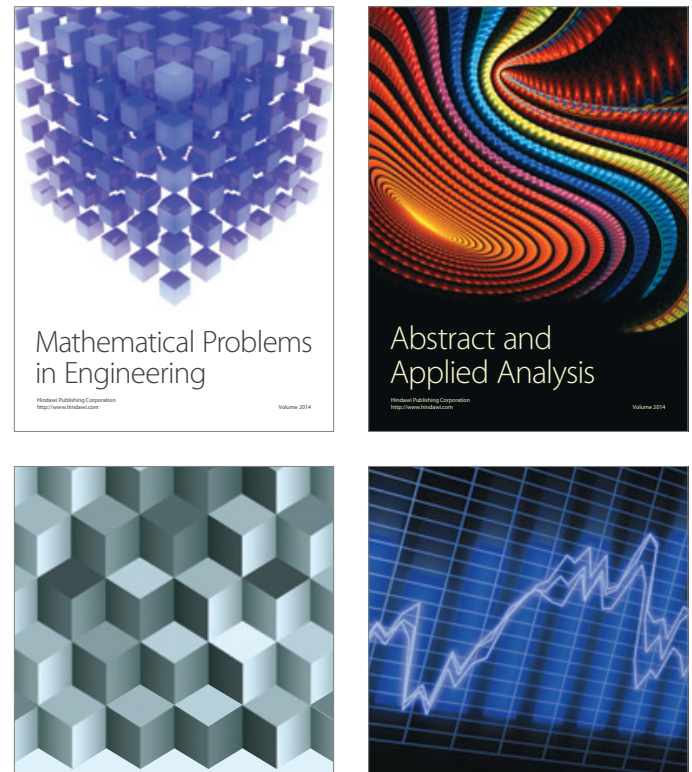

Journal of

Function Spaces

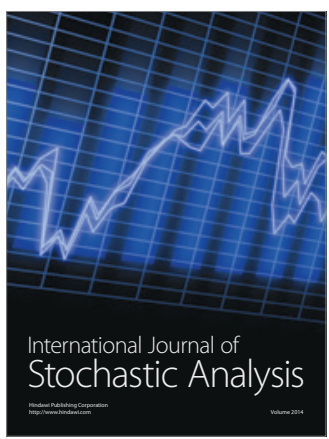

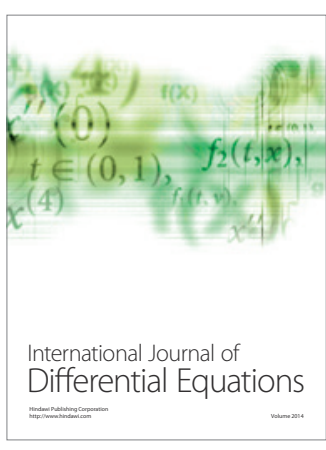
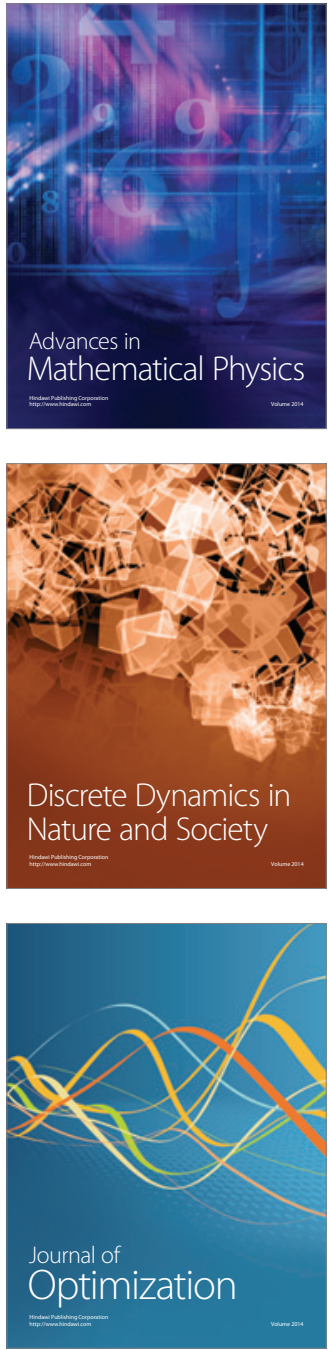